\title{
Heritability of Latent Period Estimated from Wild-Type and Selected Populations of Puccinia triticina
}

\author{
Jeffrey S. Lehman and Gregory Shaner
}

First author: Otterbein College, Department of Life and Earth Sciences, 155 West Main Street, Westerville, OH 43081; and second author: Department of Botany and Plant Pathology, Purdue University, 915 West State Street, West Lafayette, IN 47907-1155. Accepted for publication 28 February 2007.

\begin{abstract}
Lehman, J. S., and Shaner, G. 2007. Heritability of latent period estimated from wild-type and selected populations of Puccinia triticina. Phytopathology 97:1022-1029.

Durability of partially resistant wheat cultivars to wheat leaf rust depends on the amount of genetic variation in parasitic fitness within populations of the pathogen Puccinia triticina. To assess the durability of partial resistance, selection experiments were used to explore quantitative variation in parasitic fitness of $P$. triticina. Fungal populations 881-WT and 882-WT were selected for shortened latent period on partially resistant cvs. CI 13277 and Sw 72469-6 for multiple generations. Fitness components were measured for wild-type and selected fungal populations. Responses to selection and selection differentials were calculated,

period, that could provide greater fitness in nature. Generally, more cycles of selection had greater effects on fitness. In particular cases, selected populations on a partially resistant cultivar had values for latent period, uredinium area, and sporulation no different from those of a susceptible host-pathogen combination. Heritabilities of latent period of populations 881-WT and 882-WT on CI 13227 or populations 881-WT and 882-WT on $\mathrm{Sw} 72469-6$ ranged from 0.65 to 0.76 and 0.17 to 0.24 , respectively. Our results suggest the variation to overcome quantitative host resistance exists in extant populations of $P$. triticina. In addition, because more of the variation in latent period for populations of $P$. triticina on CI 13227 was genetic than for populations on Sw 72469-6, CI 13227 is likely to be more vulnerable to pathogen adaptation despite its exceptionally long latent period.
\end{abstract} and broad-sense, realized heritabilities for latent period were estimated for wild-type fungal populations on CI 13227 and on Sw 72469-6. Selected populations had fitness characteristics, not limited to latent
Additional keywords: Triticum aestivum.
Adaptation of pathogen populations toward partially resistant cultivars is dependent upon the presence of quantitative genetic variation in parasitic fitness. Fisher's fundamental theorem of natural selection states, "The rate of increase in fitness of any organism at any time is equal to its genetic variance in fitness at that time" (10). In phytopathological literature, the longevity of host resistance is regarded as being determined by the amount of genetic variation within pathogen populations $(27,28)$ or what Eenink (6) refers to as the "genetic potential" of the pathogen populations. McDonald and Linde (26) propose that the evolutionary potential of a pathogen population is reflected in its population genetic structure (i.e., the amount and distribution of genetic diversity within and among populations). They state that, in spite of low genetic recombination, asexual rust pathogens like Puccinia triticina pose medium risk of pathogen adaptation because of long-distance genotype flow. Such statements emphasize the need to quantify variation for pathogen populations on quantitatively resistant host cultivars. Knowledge of genetic variation should aid in predicting relative durability of quantitative resistance.

Numerous studies report that the fungus $P$. triticina on partially resistant wheat cultivars has a longer latent period than on susceptible cultivars $(30,32,38,39)$, produces smaller uredinia containing fewer spores $(30,32,36,39)$, and often has a reduced infection frequency $(30,32,38,39)$. The inheritance in wheat of partial resistance toward $P$. triticina and the heritability of components of host resistance also have been explored $(2-4,11,15,20-23,37)$. In con-

Corresponding author: J. S. Lehman; E-mail address: jlehman@otterbein.edu

doi:10.1094/PHYTO-97-8-1022

(c) 2007 The American Phytopathological Society trast, few studies have explored the corresponding variation in the pathogen. It is largely unknown whether variation in populations of $P$. triticina exists for all measurable components of parasitic fitness or to what extent the variation is heritable. Even for other foliar pathogens of plants, few studies have attempted to address variation in parasitic fitness. Those studies that have report that components of parasitic fitness show continuous variation on host cultivars and are, for the most part, heritable $(7,12,13,18,19$, 24,25). Observations from these studies suggest that natural selection should favor more fit pathogen isolates, and pathogen populations may overcome the partial resistance of host cultivars.

To explore quantitative variation in parasitic fitness in plantpathogenic fungi, we used selection experiments. Selection experiments are studies in which strong selective forces are applied to populations in the laboratory or field over a greatly shortened evolutionary time scale (14). Although commonly used to demonstrate polygenic variation in traits of nonphytopathogenic fungi $(29,33,34,41)$, selection experiments are less frequently used in the study of quantitative variation of plant pathogens. Notable exceptions include studies by Alexander et al. (1), Kolmer and Leonard $(18,19)$, and Kolmer $(16,17)$.

With selection experiments, heritability $(\mathrm{H})$ can be estimated from the response to selection (R) with the equation $H=R / S$, where $\mathrm{S}$ is the selection differential (i.e., the difference between the mean value of the selected portion of individuals and the mean of the total population of individuals) (9). Heritability estimated in this manner is called the realized heritability because it is primarily a description of the response. Typically, realized heritability is a measure of narrow-sense heritability (i.e., the ratio of the additive genetic variance to total phenotypic variance). Because $P$. triticina is asexual in North America and intra- and interlocus interactions cannot be separated from additive genetic 
variation, the heritability of parasitic fitness components of $P$. triticina calculated from selection experiments estimates realized, broad-sense heritability (i.e., the proportion of the total phenotypic variation that is heritable and relevant to discussions of pathogen adaptation).

Previously, we observed considerable variation in latent period among single-uredinial isolates of $P$. triticina on partially resistant cultivars (24). In simulated epidemics, isolates with short latent periods caused 2 to 2.5 times more disease than isolates with long latent periods and overcame 13 to $35 \%$ of the resistance in four partially resistant cultivars tested. Heritability estimated from analysis of variance of this variation ranged from 0.28 to 0.76 for two measures of latent period and, in general, suggests that a moderate portion of the variation in latent period is genetic and, presumably, selectable. We also observed that populations of $P$. triticina responded to selection for shortened latent period; selected populations had shorter latent periods and had larger, faster-growing uredinia than wild-type populations (25). Because the degree of selection was not quantified, we could not calculate a realized, broad-sense heritability. The objective of this study was to determine if selection for shortened latent period of wildtype populations of $P$. triticina altered their fitness on partially resistant cultivars and to quantify the selection differential and response to selection so that the heritability of latent period of wildtype populations of $P$. triticina on different partially resistant cultivars could be estimated. This current study differs from our previous studies $(24,25)$ in that (i) selection was conducted on two partially resistant cultivars of distinct genetic background with greater intensity and (ii) the degree of selection was quantified, enabling us to estimate realized, broad-sense heritability.

\section{MATERIALS AND METHODS}

Wild-type populations of $\boldsymbol{P}$. triticina. Wild-type populations of $P$. triticina, populations $881-\mathrm{WT}$ and $882-\mathrm{WT}$, were collected in the fall of 1987 from naturally infected wheat plants growing near Tipton and Romney, IN, respectively. From plants at each site, $\approx 50$ severely infected leaves were collected, and spores were vacuumed from their surfaces. Initial quantities of spores were increased for one generation on susceptible wheat $\mathrm{cv}$. Monon (CI 13278) and stored at $-80^{\circ} \mathrm{C}$ until used in selection experiments.

Cultivars of Triticum aestivum. Wild-type populations were selected for shortened latent period on partially resistant cvs. CI 13227 (Wabash/American Banner//Klein Anniversario) and Sw 72469-6 (Strampelli/69D-3607//Chokwang). Cv. CI 13227 has been shown to have four genes with epistatic effects that control long latent period (37) whereas cv. Sw 72469-6 has been shown to have two genes (21). Host seedlings of these cultivars were vernalized at $3^{\circ} \mathrm{C}$ for 6 to 8 weeks under $12 \mathrm{~h}$ /day of fluorescent light. After vernalization, seedlings were transplanted individually into $400-\mathrm{ml}$ plastic pots containing a soil/peat mixture and were grown in the greenhouse. Natural daylight was supplemented with incandescent and fluorescent light for $16 \mathrm{~h} /$ day $(\approx 200 \mu \mathrm{mol}$ quanta $\mathrm{m}^{-2} \mathrm{~s}^{-1}$ ) from time of transplanting to maturity. Day and night temperatures were 20 to 24 and 17 to $20^{\circ} \mathrm{C}$, respectively.

Selection procedure. Adaxial surfaces of 20 to 25 newly emerged flag leaves (the uppermost leaf) of cvs. CI 13227 or Sw 72469-6 were inoculated with urediniospores suspended in Soltrol 170 light mineral oil (Philips Petroleum Co., Hennepin, OK) or water at a rate of $1 \mathrm{mg}$ of spores per milliliter of oil/water. Inoculated plants were misted with water-Tween 20 (one drop of Tween 20 per liter of $\mathrm{H}_{2} \mathrm{O}$ ) and placed in a moist chamber at 20 to $22^{\circ} \mathrm{C}$ for 12 to $14 \mathrm{~h}$. Uredinia typically erupt from leaf surfaces of CI 13227 or Sw 72469-6 over the interval of 6 to 18 days after inoculation; the plot of the number of uredinia that erupt each day against day after inoculation yields a bell-shaped curve slightly skewed to the right. Because of this distribution of latent periods for individual uredinia, urediniospores from early erupting uredinia could be collected separately from those of later erupting uredinia (i.e., truncation selection). After a portion of the uredinia began to erupt and sporulate, spores were vacuumed from the adaxial leaf surface with a cyclone collector (43) attached to a vacuum pump. Spores, collected in bulk, were chosen strictly based on the latent period of the uredinia that produced them. The truncated population of spores was the product of one cycle of selection for shortened latent period and either was stored at $-80^{\circ} \mathrm{C}$ or used to inoculate new flag leaves of the same partially resistant cultivar for multiple cycles of selection.

Selected populations of $\boldsymbol{P}$. triticina. In total, 11 populations were derived from 881-WT and 882-WT reared on CI 13227 or Sw 72469-6 by repeating multiple cycles of selection for shortened latent period (i.e., truncating the distribution of latent periods at 8 to 14 days after inoculation) (Table 1). Four of these, populations $881-\mathrm{C} 3$ and $881-\mathrm{S} 4$ selected from 881 -WT and populations $882-\mathrm{C} 1$ and $882-\mathrm{S} 3$ selected from $882-\mathrm{WT}$, were tested for changes in parasitic fitness along with their respective wild-type progenitors. In this article, studies measuring changes in fitness of populations selected on CI 13227 are collectively referred to as series $\mathrm{C}$ experiments (i.e., the series of experiment measuring populations selected on CI 13227). Studies measuring populations selected on Sw 72469-6 are collectively referred to as series S.

Measurement of components of parasitic fitness and host resistance. Mean latent period, uredinium area, and cumulative urediniospore production were measured for populations 881-WT, 881-C3, 882-WT, and 882-C1 on partially resistant cvs. CI 13227 and L 574-1 (Wakeland/Blueboy) and susceptible cv. Monon (series C). Only latent period was measured for populations 881WT, 881-S4, 882-WT, and 882-S3 on partially resistant CI 13227 and Sw 72469-6 and on susceptible Monon (series S).

Flag leaves of cultivars were inoculated with individual fungal populations with a spore settling tower (8). We estimated a deposition of two to three viable spores per square millimeter of flag leaf based on spore density and percent germination observed on $1.5 \%$ water agar plates. Inoculated plants were misted with waterTween 20 (one drop of Tween 20 per liter of $\mathrm{H}_{2} \mathrm{O}$ ) and placed in a moist chamber at 20 to $22^{\circ} \mathrm{C}$ for 12 to $14 \mathrm{~h}$. On days 6 to 20 after inoculation, uredinia which had erupted from the adaxial surface of the middle 3 to $5 \mathrm{~cm}$ of the flag leaf at a density of $\approx 0.2$ pustules $/ \mathrm{mm}^{2}$ were counted. Mean latent period (MLP), the

TABLE 1. List and description (origin, range of latent periods, and day of truncation during selection) of wild-type and selected populations of Puccinia triticina

\begin{tabular}{|c|c|c|c|c|}
\hline \multirow[b]{2}{*}{ Population } & \multirow[b]{2}{*}{ Origin $y$} & \multicolumn{2}{|c|}{$\begin{array}{c}\text { Range of } \\
\text { latent periods on }\end{array}$} & \multirow[b]{2}{*}{ Trunc $^{\mathrm{Z}}$} \\
\hline & & $\mathrm{CI}$ & Sw & \\
\hline 881-WT & Tipton, IN & $6-17$ & $6-18$ & - \\
\hline $881-\mathrm{C} 1$ & 881-WT on CI 13227 & - & - & 14 \\
\hline $881-\mathrm{C} 2$ & $881-\mathrm{C} 1$ in CI 13227 & - & - & 10 \\
\hline $881-\mathrm{C} 3$ & $881-\mathrm{C} 2$ on CI 13227 & $6-10$ & - & 8 \\
\hline 882-WT & Romney, IN & $6-17$ & $6-17$ & - \\
\hline $882-\mathrm{C} 1$ & 882-WT on CI 13227 & $6-16$ & - & 14 \\
\hline $881-\mathrm{S} 1$ & 881-WT on Sw 72469-6 & - & - & 14 \\
\hline $881-\mathrm{S} 2$ & 881-S1 on Sw 72469-6 & - & - & 11 \\
\hline $881-\mathrm{S} 3$ & $881-\mathrm{S} 2$ on $\mathrm{Sw} 72469-6$ & - & - & 9 \\
\hline $881-\mathrm{S} 4$ & 881-S3 on Sw 72469-6 & - & $6-13$ & 9 \\
\hline $882-\mathrm{S} 1$ & 882-WT on Sw 72469-6 & - & - & 14 \\
\hline $882-\mathrm{S} 2$ & 882-S1 on Sw 72469-6 & - & - & 11 \\
\hline $882-\mathrm{S} 3$ & $882-\mathrm{S} 2$ on $\mathrm{Sw} 72469-6$ & - & $6-14$ & 9 \\
\hline
\end{tabular}

${ }^{x}$ First and last day on which uredinia erupt from adaxial leaf surface of cv. CI 13227 or Sw 72469-6.

y Location of collection site or the progenitor population.

${ }^{\mathrm{z}}$ Day of truncation; day after inoculation on which selection occurred. Spores produced after this day were excluded from the selected portion of individuals. Spores produced before this day were collected in bulk and increased on susceptible cv. Monon. The resultant progeny were collectively designated as the selected population. 
weighted average time for uredinia to erupt, was calculated from uredinial counts from the following equation:

$$
\mathrm{MLP}=\sum_{i=0}^{n} P_{i} t_{i}
$$

in which $P_{i}$ is the proportion of uredinia relative to the final number of uredinia that appear on the $i$ th day after inoculation, $t_{i}$ is the $i$ th day after inoculation, and $n$ is the number of days after inoculation when all uredinia have appeared.

After all uredinia had erupted, the length (L) and width (W) of 10 randomly selected uredinia were measured, and uredinium area (UA) was calculated as $\mathrm{UA}=(\mathrm{L})(\mathrm{W})(3.14) / 4$.

On days $9,12,15$, and 18, spores were vacuumed in bulk from a known number of uredinia. Spores were suspended in $10 \mathrm{ml}$ of phosphate-buffered saline with $0.5 \%$ Tween 20 , and a $500-\mathrm{ml}$ sample of spores was counted with a Model FN Coulter Counter (Coulter Electronic Inc. Hialeah, FL). The average number of spores per uredinium was calculated by dividing the total spore production by the number of uredinia present on a given collection day. Means for the four collection days were totaled to calculate cumulative urediniospore production.

Experimental design and statistical analysis. The experimental design was a randomized complete block design with main effects of two experiments, six replications, five cultivars, and four fungal populations. The linear model for the analysis was $\mathrm{Y}_{\mathrm{ijk} l}=\mu+\mathrm{L}_{\mathrm{i}}+\mathrm{R}_{(\mathrm{i}) \mathrm{j}}+\mathrm{C}_{\mathrm{k}}+\mathrm{LC}_{\mathrm{ik}}+\mathrm{P}_{\mathrm{l}}+\mathrm{LP}_{\mathrm{il}}+\mathrm{CP}_{\mathrm{kl}}+\mathrm{LCP}_{\mathrm{ikl}}+\mathrm{E}_{(\mathrm{i}) \mathrm{jk} l}$, where $\mathrm{Y}_{\mathrm{ijkl}}=$ response of the $i j k l$ th individual subunit; $\mu=$ overall mean; $\mathrm{L}_{\mathrm{i}}=$ effect of $i$ th experiment, $\mathrm{i}=1,2$ experiments; $\mathrm{R}_{(\mathrm{i}) \mathrm{j}}=$ effect of $j$ th block nested within experiments; $\mathrm{j}=1 \ldots 6$ replications; $\mathrm{C}_{\mathrm{k}}=$ effect of $k$ th wheat cultivar; $\mathrm{k}=1 \ldots .5$ cultivars; $\mathrm{LC}_{\mathrm{ik}}=$ interaction effect of the $i$ th experiment and $k$ th cultivar; $\mathrm{P}_{1}$ $=$ effect of $p$ th population, $1=1 \ldots 4$ populations of $P$. triticina; $\mathrm{LP}_{\mathrm{il}}=$ interaction effect of the $i$ th experiment and $l$ th population; $\mathrm{CP}_{\mathrm{kl}}=$ interaction effect of the $k$ th cultivar and $l$ th population; $\mathrm{LCP}_{\mathrm{ikl}}=$ interaction effect of the $i$ th experiment, $k$ th cultivar, and $l$ th population; and $\mathrm{E}_{(\mathrm{i}) \mathrm{jk} l}=$ random effect of the $j k l$ th subunit in the $i$ th experiment.

A $\log _{10}$ transformation of MLP and cumulative urediniospore production effectively eliminated the association between mean and variance that existed for untransformed statistics (42). Data for uredinium area were not transformed. Student-Newman-Keuls tests $(\alpha=0.05)$ were used to separate means for cultivars, populations, and the interaction between cultivar and population (42).

Estimation of heritabilities. Regression analysis was used to estimate the realized, broad-sense heritabilities of MLP for population 881-WT on CI 13227 and populations 881-WT and 882WT on Sw 72469-6. Because 882-WT was selected on CI 13227 for only one generation (i.e., there was a total of only two populations), regression analysis was not used to estimate heritability for this cultivar-population combination. Rather, heritability was calculated from the response or actual change in MLP divided by the selection differential (i.e., the deviation of the mean phenotypic value of the selected individuals from the mean phenotypic value of the intact parental population) (9).

Heritabilities were estimated from the slopes of the MLPs of wild-type and selected populations regressed onto the cumulative deviation in latent period for the portion of individuals selected from the progenitor population (i.e., the cumulative response to selection regressed onto the cumulative selection differential) (9). The MLPs of the wild-type and most highly selected population (e.g., 881-WT and 881-C3) were measured experimentally based on the distribution of latent periods for erupting uredinia, as previously described. The latent periods for intermediate populations (e.g., 881-C1 and 882-C2) were estimated by gradually transfiguring or "morphing" the distribution of latent periods for uredinia of wild-type populations into that of the most selected population. To do this, we scaled the MLP of the known populations to zero and took a weighted average of the frequency of newly erupted uredinia of the two known populations for days 6 to 20 after inoculation. For example, the estimated frequency of newly erupted uredinia of population $881-\mathrm{C} 1$ on a particular day was $\mathrm{P}_{\mathrm{Cli}}=(0.667)\left(\mathrm{P}_{\mathrm{WTi}}\right)+(0.333)\left(\mathrm{P}_{\mathrm{C} 3 \mathrm{i}}\right)$, where $\mathrm{P}_{\mathrm{Cli}}, \mathrm{P}_{\mathrm{WTi}}$, and $\mathrm{P}_{\mathrm{C} 3 \mathrm{i}}$ are the proportion of uredinia relative to the final number of uredinia that appear on the $i$ th day in population $881-\mathrm{C} 1,881$ WT, and 881-C3, respectively. The weights 0.667 and 0.333 were switched for estimating the latent period distributions of population 881-C2. After the distribution of latent periods for uredinia were estimated, the MLPs for populations intermediate to the wild-type and most selected populations were calculated as a weighted mean average and later used as our dependent variable in regression analyses.

The independent variable in our regression was the cumulative selection differential based in the deviation of the mean phenotypic value for latent period of uredinia selected in our truncation selection procedure from the progenitor population (9). Because our selection procedure truncated spore production of the uredinia which had erupted before the day of truncation in addition to latent period (i.e., early-erupting uredinia produced more spores and their latent period should be weighted correspondingly in the calculation of MLP), we had to consider in the calculations of the MLP of individuals selected from the intact population the relative number of spores produced by uredinia. Thus, the MLP of the truncated population (i.e., selected individuals) was estimated from the weighted average latent period for individuals where the weights are a function of the relative amount of urediniospores produced before the day of truncation, in addition to the frequency of uredinia. This was similar to weighting the selection differential as described by Falconer and Mackay (9). The MLP of truncated populations was calculated from the following equation:

$$
\left.M L P(\text { selectedindividuals })=\left[\left(\sum_{i=0}^{n} w_{i} P_{i} t_{i}\right)\right] / \sum_{i=0}^{n} P_{i} t_{i}\right]
$$

where which $\mathrm{w}_{\mathrm{i}}$ is the relative proportion of urediniospores produced by a uredinium from time of eruption to the day of truncation, $P_{i}$ is the proportion of uredinia relative to the total number of uredinia that appear on the $i$ th day after inoculation, $t_{i}$ is the $i$ th day after inoculation, and $n$ is truncation point or day after inoculation when selection occurred.

The selection differential was calculated as the difference between the MLP of the total, intact population and the MLP of the selected individuals. The MLPs for wild-type and selected populations were regressed onto the cumulative selection differential, and slopes (i.e., heritabilities) of the regression lines were calculated. $t$ tests $(\alpha=0.05)$ were used to compare heritabilities estimated from populations reared on CI 13227 with those from populations reared on $\mathrm{Sw} 72469-6$ (42).

\section{RESULTS}

Populations and cultivars responded consistently across experiments; interactions between effects of cultivar or population with experiment were typically nonsignificant. When exceptions did occur, they were due to magnitudinal differences of the fitness parameter for population or cultivars within experiments and not due to changes in rank. For example, the experiment-cultivar interaction for selected series $\mathrm{C}$ was significant due to a magnitudinal increase in log MLP of CI 13227 in one experiment compared with the other; the relative rank for cultivars within experiments remained unchanged.

Latent period. The effect of cultivar was a significant source of variation for series $\mathrm{C}(P=0.0057)$. For series $\mathrm{S}$, the $P$ value for the effect of cultivar was 0.069 . In the former, all cultivars differed for latent period, and values for Monon and CI 13227 were the lowest and highest, respectively (Table 2). In series S, only cv. 
CI 13227 had a latent period longer than that of Monon; the latent period of Sw 72469-6 and Monon were not significantly different (Table 2). Latent periods of L 574-1 and CI 13227 were 1.8 and 3.7 days longer than that of Monon in series $\mathrm{C}$. In series $\mathrm{S}$, the latent period of CI 13227 was 5.4 days longer.

For series $\mathrm{C}$, the main effect of population was significant $(P=$ $0.0042)$ and, averaged across experiments, replications, and cultivars, selected population 881-C3 had a latent period 2.3 days shorter than its wild-type progenitor (Table 2). For series $\mathrm{S}$, the $P$ value was 0.092 for the main effect of population. Averaged across experiments, replications, and cultivars, the latent period of 881-S4 was significantly shorter (0.75 days) than that of 881-WT (Table 2). Differences between 882-S3 and 882-WT were nonsignificant.

The interaction of cultivar-fungal population was highly significant for selected series $C(P=0.0009)$. This was largely due to 2.2- or 4.8-day decreases in the MLP of 881-C3 on L 574-1 and CI 13227, respectively, compared with values for the wild-type progenitor (Fig. 1). On both cultivars, population 881-C3 was no different for latent period than 881-WT on susceptible Monon. In contrast, selected population $882-\mathrm{C} 1$ had a shorter latent period than 882-WT on CI 13227 only, and the MLP of 882-C1 on CI 13227 was still significantly longer than $882-\mathrm{C} 1$ on Monon. Differences in latent period between populations on Monon were nonsignificant. For series S, the MLPs for populations 881-S4 and $882-\mathrm{S} 3$ on $\mathrm{Sw} 72469-6$ were 1.8 and 0.9 days shorter, respectively, than latent periods of populations $881-\mathrm{WT}$ and $882-\mathrm{WT}$ on this cultivar (Fig. 2). Despite this reduction, population 882-S3 had a longer latent period on Sw 72469-6 than it did on Monon; population 881 -S4 did not. There were no differences in latent period among populations on cvs. CI 13227 or Monon.

TABLE 2. Mean latent period, (MLP) uredinium area (Area), and cumulative number of spores per uredinium (Number) of wild-type and selected populations of Puccinia triticina on susceptible and partially resistant cultivars of Triticum aestivum

\begin{tabular}{|c|c|c|c|c|}
\hline \multirow[b]{3}{*}{ Wheat, fungus ${ }^{w}$} & \multicolumn{4}{|c|}{ Selection on } \\
\hline & \multicolumn{3}{|c|}{ CI 13227} & \multirow{2}{*}{$\frac{\text { Sw 72469-6 }}{\text { MLP (days) }^{x}}$} \\
\hline & $\operatorname{MLP}(\text { days })^{\mathrm{x}}$ & Area $\left(\mathrm{mm}^{2}\right)^{y}$ & Number $^{z}$ & \\
\hline \multicolumn{5}{|l|}{ Wheat } \\
\hline Monon & $6.8 \mathrm{a}$ & $0.22 \mathrm{c}$ & $1,616 \mathrm{~b}$ & $7.7 \mathrm{a}$ \\
\hline L-574-1 & $8.6 \mathrm{~b}$ & $0.20 \mathrm{~b}$ & $1,279 \mathrm{ab}$ & \\
\hline Sw 72469-6 & & & & $9.5 \mathrm{ab}$ \\
\hline CI 13227 & $10.5 \mathrm{c}$ & $0.17 \mathrm{a}$ & $1,016 \mathrm{a}$ & $13.1 \mathrm{~b}$ \\
\hline \multicolumn{5}{|l|}{ Fungus } \\
\hline 881-WT & $9.2 \mathrm{~b}$ & $0.19 \mathrm{a}$ & $1,349 \mathrm{a}$ & $10.5 \mathrm{~b}$ \\
\hline 881-C3 & $6.9 \mathrm{a}$ & $0.24 \mathrm{~b}$ & $1,412 \mathrm{~b}$ & \\
\hline 882-WT & $9.4 \mathrm{~b}$ & $0.21 \mathrm{ab}$ & $1,231 \mathrm{a}$ & $10.3 \mathrm{ab}$ \\
\hline 882-C1 & $8.9 \mathrm{~b}$ & $0.18 \mathrm{a}$ & $1,226 \mathrm{a}$ & \\
\hline 881-S4 & $\ldots$ & $\ldots$ & $\ldots$ & $9.7 \mathrm{a}$ \\
\hline $882-S 3$ & $\ldots$ & $\ldots$ & $\ldots$ & $10.0 \mathrm{ab}$ \\
\hline
\end{tabular}

${ }^{\mathrm{w}}$ Wheat cultivar or fungal population.

${ }^{x}$ MLP is the weighted mean number of days from infection to the production of inoculum. Each value is the mean of two experiments with six replications averaged across populations of $P$. triticina or cultivars of wheat. Means for wheat cultivars or fungal populations with a letter in common do not differ significantly for log-transformed data according to Student-Newman-Keuls mean separation tests $(\alpha=0.05)$.

${ }^{y}$ Uredinium area was calculated as $(\mathrm{L})(\mathrm{W})(3.14) / 4$, where $\mathrm{L}$ and $\mathrm{W}$ are uredinium length and width, respectively. Each value is the mean of two experiments with six replications averaged across populations of $P$. triticina or cultivars of wheat. Means for wheat cultivars or fungal populations with a letter in common do not differ significantly according to Student-NewmanKeuls mean separation tests $(\alpha=0.05)$.

${ }^{\mathrm{z}}$ Cumulative urediniospore production is the cumulative number of spores per uredinium produced on days $9,12,15$, and 18 after inoculation. Values are the mean of two experiments with six replications averaged across populations of P. triticina or cultivars of wheat. Means for wheat cultivars or fungal populations with a letter in common do not differ significantly for log-transformed data according to Student-Newman-Keuls tests $(\alpha=0.05)$.
Uredinium area and sporulation. Uredinium area and cumulative sporulation were measured for series $\mathrm{C}$ only. The main effect of cultivar was significant for uredinium size $(P=0.0039)$, and the means for all cultivars differed (Table 2). Uredinia on CI 13227 and L 574-1 were 78 and 92\% smaller than uredinia on Monon. Population 881-C3 produced significantly larger uredinia averaged across experiments, replications, and cultivars than population 881-WT (Table 2). Uredinia of population 881-C3 on CI 13227 were $73 \%$ larger than those produced by $881-W T$ and no different in size from those produced on susceptible cultivars (Fig. 3). In contrast, the uredinia of population 882-C1 were no different in size than those of 882-WT averaged across experiments and replications (Fig. 3).

The $P$ value for the effect of cultivars on cumulative urediniospore production was 0.10 . Averaged across experiment, replications, and populations, the fungus produced more spores on cv. Monon than on CI 13227 but not on L 574-1 (Table 2). Similarly, the $P$ value for the effect of population on cumulative urediniospore production was $P=0.09$. Averaged across experiment, replications, and cultivar, the mean spore production of population 881-C3 was higher than that of population 881-WT. In contrast, the number of spores produced for populations $882-\mathrm{C} 1$

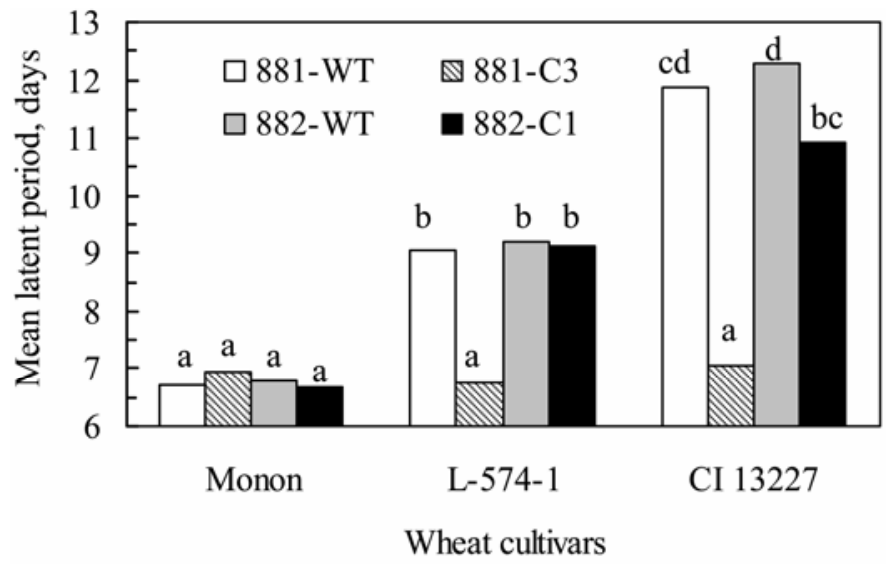

Fig. 1. Mean latent periods of Puccinia triticina populations 881-WT, 881-C3, 882-WT, and 882-C1 on partially resistant cvs. CI 13227 and L-574-1 and susceptible cv. Monon. Mean latent period is the weighted mean number of days from infection to the production of inoculum. Each bar is the mean of two experiments with six replications. Cultivar-population means with a letter in common above the bar do not differ significantly for log transformed data according to Student-Newman-Keuls test.

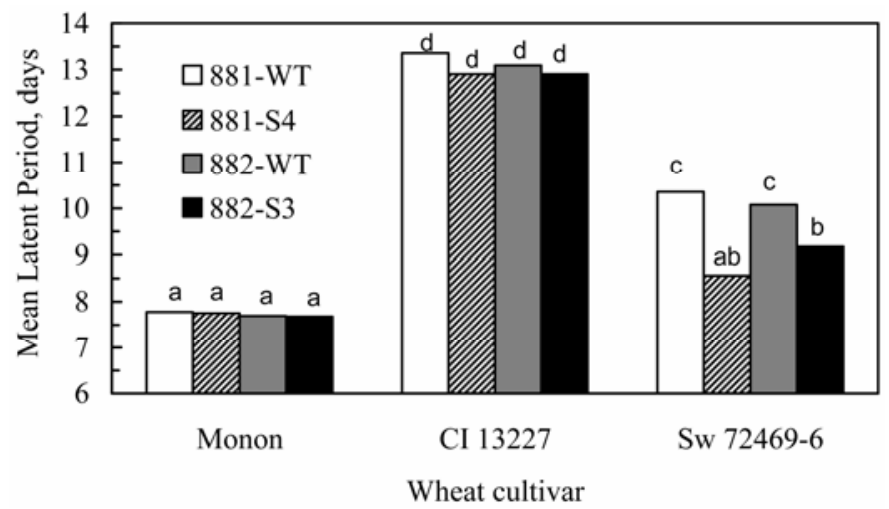

Fig. 2. Mean latent periods of Puccinia triticina populations 881-WT, 881-S4, 882-WT, and 882-S3 on partially resistant wheat cvs. Sw 72469-6 and CI 13227 and susceptible cv. Monon. Mean latent period is the weighted mean number of days from infection to the production of inoculum. Each bar is the mean of two experiments with six replications. Cultivar-population means with a letter in common above the bar do not differ significantly for log transformed data according to Student-Newman-Keuls test. 
was no different from that produced by population 881-WT (Table 2).

The interaction between cultivar and population was significant $(P=0.015)$. Means for populations only on CI 13227 and Monon differed for cumulative urediniospore production (Fig. 4). On CI 13227 , both selected populations $881-\mathrm{C} 3$ and $882-\mathrm{C} 1$ produced 2 and 1.4 times more spores, respectively, than their wild-type progenitors. These differences were largely due to spores produced on days 9, 12, and 15 after inoculation for 881-WT and 881-C3 and on day 12 after inoculation for 882-WT and 882-C1 (Table 3). On Monon, the trend was reversed; population 881-C3 produced $57 \%$ fewer spores than $881-W T$ (Fig. 4). There were no differences between $882-\mathrm{C} 1$ and $882-\mathrm{WT}$ for cumulative urediniospore production on Monon.

Heritability estimates of MLP. Natural selection operates on pathogen populations whenever the fitnesses of fungal genotypes differ. Hence, knowledge of quantitative genetic variation in parasitic fitness is essential to understanding adaptation of populations of $P$. triticina toward partially resistant cultivars. To quantify genetic variation for latent period, we calculated broad-sense

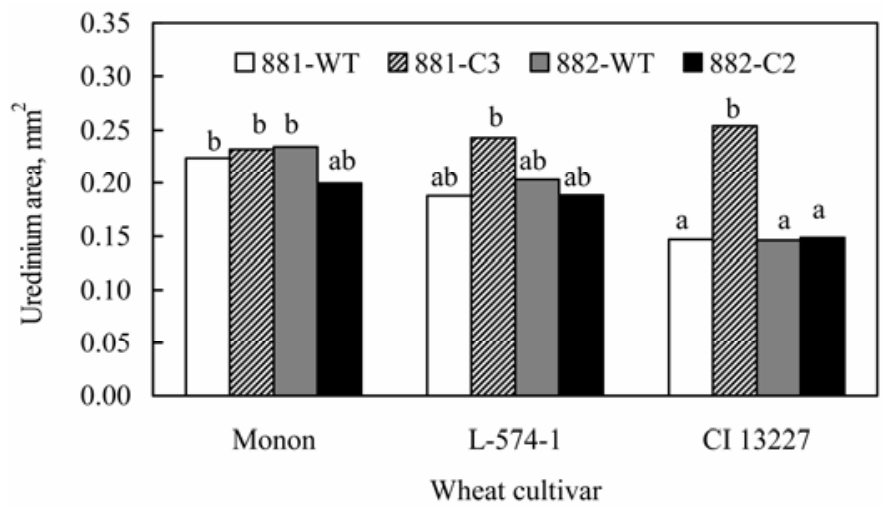

Fig. 3. Uredinium area (UA) of Puccinia triticina populations 881-WT, 881C3, 882-WT, and 882-C1 on partially resistant wheat cvs. CI 13227 and L574-1 and susceptible cv. Monon. Uredinium area is calculated from the equation $\mathrm{UA}=(\mathrm{L})(\mathrm{W})(3.14) / 4$, where $\mathrm{L}$ and $\mathrm{W}$ are uredinium length and width, respectively. Each bar is the mean of two experiments with six replications. Cultivar-population means with a letter in common above the bar do not differ significantly according to Student-Newman-Keuls test.

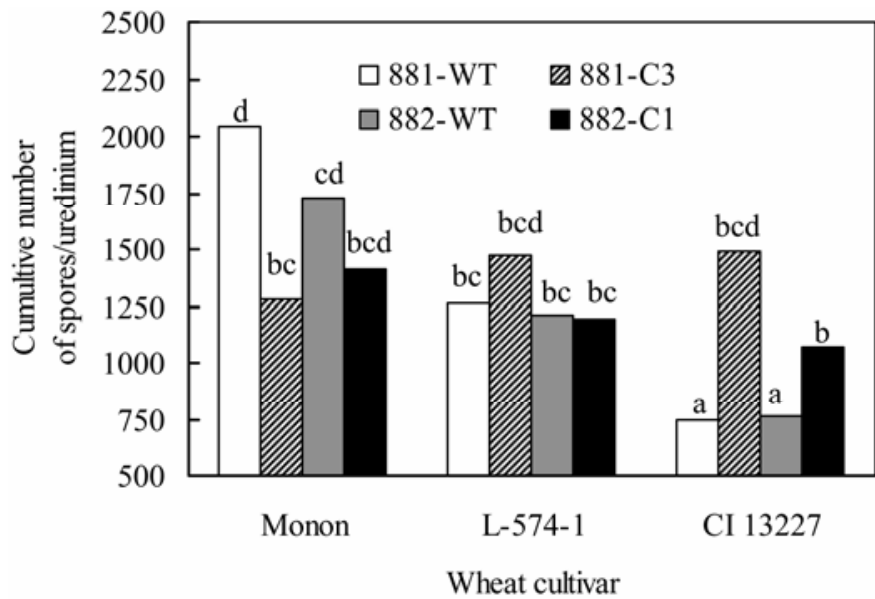

Fig. 4. Cumulative urediniospore production of Puccinia triticina populations 881-WT, 881-C3, 882-WT, and 882-C1 on partially resistant cvs. CI 13227 and L-574-1 and susceptible cv. Monon. Cumulative urediniospore production is the cumulative number of spores per uredinium produced on days $9,12,15$, and 18 after inoculation. Each bar is the mean of two experiments with six replications. Cultivar-population means with a letter in common above the bar do not differ significantly for log transformed data according to StudentNewman-Keuls test. heritability, the ratio of genetic variation to phenotypic variation based on the response to selection. Because P. triticina in North America does not undergo sexual recombination, all the variation partitioned as genetic should be passed on to asexual offspring (i.e., dominance and epistasis which upwardly bias estimates of broad-sense heritability relative to narrow-sense heritability are not pertinent in the absence of recombination). Broad-sense heritabilities of latent period should represent the variation in populations of $P$. triticina that influences adaptation toward partially resistant cultivars for this trait.

Coefficients of determination for regression lines from which heritabilities were calculated were $\approx 0.99$ (Fig. 5). In general, heritability estimates for MLP were higher for populations of P. triticina on CI 13227 than on $\mathrm{Sw}$ 72469-6. Estimates for populations 881-WT and 882-WT on Sw 72469-6 were 0.24 and 0.17 , respectively. Estimates for population 881-WT on CI 13227 was 0.76 and significantly higher than either population on $\mathrm{Sw}$ 72469-6. The realized heritability for 882-WT on CI 13227 calculated from the response and selection differential from one generation of selection, not the regression equation, was 0.65 .

\section{DISCUSSION}

Components of host resistance are measured routinely for wheat cultivars with quantitative resistance toward $P$. triticina. Corresponding attributes of parasitic fitness in the pathogen have received considerably less attention despite their equally im-

TABLE 3. Sporulation on days $9,12,15$, and 18 after inoculation of adult plants of wheat cv. CI 13227 inoculated with wild-type populations of Puccinia triticina and populations selected for shortened latent period on CI $13227^{7}$

\begin{tabular}{lcccc}
\hline & \multicolumn{4}{c}{ Days after inoculation } \\
\cline { 2 - 5 } Population & 9 & 12 & 15 & 18 \\
\hline $881-W T$ & $146 \mathrm{a}$ & $182 \mathrm{a}$ & $246 \mathrm{a}$ & $221 \mathrm{a}$ \\
$881-\mathrm{C} 3$ & $427 \mathrm{c}$ & $484 \mathrm{c}$ & $419 \mathrm{c}$ & $163 \mathrm{a}$ \\
$882-W T$ & $256 \mathrm{~b}$ & $146 \mathrm{a}$ & $258 \mathrm{ab}$ & $188 \mathrm{a}$ \\
$882-\mathrm{C} 1$ & $297 \mathrm{~b}$ & $208 \mathrm{~b}$ & $353 \mathrm{~b}$ & $212 \mathrm{a}$
\end{tabular}

${ }^{\mathrm{z}}$ Sporulation is the number of spores per uredinium produced on days 9,12 , 15 , or 18 after inoculation. Values are the means of two experiments with six replications. Means for populations on individual days after inoculation with a letter in common do not differ significantly for $\log _{10}$-transformed data according to Student-Newman-Keuls tests $(\alpha=0.005)$. Untransformed means are presented.

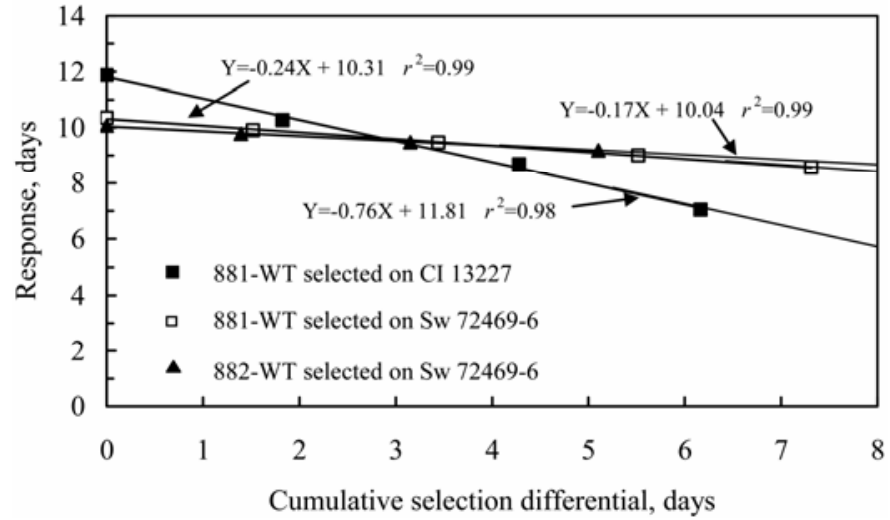

Fig. 5. Linear regression and coefficients of determination $\left(r^{2}\right)$ between the response to selection (i.e., change in mean latent period) and cumulative selection differential for wild-type populations 881-WT and 882-WT on CI 13227 and Sw 72469-6. Cumulative selection differentials (i.e., sums of individual selection differentials for each population) are presented as positive integers for conventionality of graphical presentation; they are actually negative. Heritabilities (i.e., the slopes of the lines) are also positive; the negative value reflects the change in mean latent period after selection. 
portant role in determining the rate of disease progression. Although it is generally assumed that pathogen genotypes differ for fitness, pathologists understand little about the components contributing to fitness or the genetic variation associated with these components. Because selection operates whenever the fitness of pathogen genotypes differ, knowledge of genetic variation in parasitic fitness would be useful for predicting pathogen adaptation toward host resistance.

In its broadest context, fitness is defined as the contribution an individual makes to the gene pool in the next generation (35). Fitness is a function of population size (5) and commonly is measured by the rate at which genotypes increase in frequency in the population relative to other genotypes (i.e., the Malthusian parameter) (10). In a phytopathological context, fitness is one of the many terms used to describe the ability of a pathogen to persist in nature (40).

Pathogen genotypes can be compared in monocyclic infection experiments by quantifying components of parasitic fitness (i.e., components that contribute to the reproduction of the pathogen). The genetic variation of these components and their propensity to change when pathogen populations are under selection may be the best predictor of the durability of host resistance short of the actual deployment of host resistance; high heritabilities (i.e., high additive genetic variance) and rapid, large responses of pathogen populations to selection are indicative of nondurable host resistance.

Selection altered fitness components of $P$. triticina populations. Averaged across all cultivars, populations that were the most highly selected on CI 13227 or Sw 72469-6 (i.e., populations 881C3 and 881-S4) had short latent periods and large uredinia and produced more spores. These characteristics could provide greater reproductive capacity in nature compared with wild-type fungal populations. These differences between wild-type and selected populations suggest that wild-type populations are heterogeneous with regard to parasitic fitness and that genotypes of greater fitness on partially resistant cultivars exist in wild-type populations at low frequencies. Our selection regime disfavored individuals with long latent period, a characteristic that reduces an individual's contribution to subsequent generations. In contrast, those individuals with short latent periods produce offspring earlier; hence, when selection was practiced on fungal populations, individuals with short latent periods contributed more offspring to the bulked population of spores to increase in frequency with subsequent generations.

The question arises as to whether selected populations with greater fitness averaged across all cultivars have general or specific fitness. Although the MLP, uredinium area, and sporulation of fungal populations averaged across cultivars suggests that $881-\mathrm{C} 3$ and $881-\mathrm{S} 4$ are generally more fit, these differences are the result of increased fitness on only one or two cultivars. They typically were more fit on the cultivar upon which selection occurred. With repeated cycles of selection, fitness differences became greater. In contrast, there were no differences between less intensely selected population $882-\mathrm{C} 1$ or $882-\mathrm{S} 3$ and their wild-type progenitors averaged across cultivars; yet, as described for population 881-C3 and 881-S4, there were differences in fitness between populations $882-\mathrm{C} 1$ or $882-\mathrm{S} 3$ and their respective wild-type populations on the host upon which they were selected. From these results, it appears that differences in fitness components between pathogen populations are the result of increases in specific, not general, fitness.

In addition to the fact that selected populations were more fit on the host upon which they were selected, selected population 881-C3 had reduced fitness for cumulative sporulation relative to its wild-type progenitor on the susceptible cv. Monon. Such differences suggest that there is a "cost" to increased fitness toward partially resistant CI 13227 on cultivars where it is not required. However, fitness components of selected populations of
P. triticina on the host upon which selection occurred were generally no different than the components of wild-type populations on susceptible Monon.

Selection resulted in populations of $P$. triticina overcoming the genes which control latent period in CI 13227 and Sw 72469-6. Similarly, population 881-C3 overcame all the resistance in CI 13227 for uredinium area and sporulation. Because of the important role of latent period, sporulation, and uredinium area in disease progression, disease development of population 881-C3 on CI 13227 should be greater than that of population 881-WT on CI 13227 and no different than that of population 881-WT on Monon. Disease for CI 13227 inoculated with population 881-C3 may even be greater than that for Monon inoculated with population 881-C3 because of the reduced sporulation of 881-C3 on Monon relative to the wild-type population. In previous selection experiments (25), selection improved the parasitic fitness of populations of $P$. triticina; however, in no case were the components of fitness for selected populations on partially resistant cultivars equivalent to those of wild-type fungal populations on Monon. The greater responses of selected populations in this study suggest that either selection for shortened latent period was more intense than in previous studies or that pathogen populations of the present study exhibited greater variation in latent period and, therefore, were more responsive to selection.

Although selected for shortened latent period, selected populations on CI 13227 also differed for uredinium area and cumulative sporulation, possibly because of pleiotropy or linkage. Evidence for genetic correlations between latent period and uredinium area for partially resistant cultivars has been reported $(20,21,23)$. Correlated responses between shortened latent period and increased uredinial size, uredinial growth rate, and sporulation were also observed in the selection of 851-WT on CI 13227 (25). The occurrence of correlated responses is expected because, when we were selecting primarily for short latent period, we likely selected for prolifically sporulating individuals or individuals that contributed more to the selected population of spores because of their large uredinia.

Differences in cumulative urediniospore production for wildtype and selected populations on CI 13227 were due largely to the number of spores produced on days 9, 12, and 15 after inoculation. These results and the observation that sporulation is greatest when uredinia are young and then decreases with age (44) suggest that earlier spore production periods are most likely to distinguish populations of $P$. triticina. Because collecting and counting spores is resource intensive, urediniospores should be collected in the early phase of sporulation when sporulation peaks; sporulation data from aging uredinia are not likely to distinguish populations.

In addition to being more fit on CI 13227, selected population 881-C3 also was more fit on L-574-1, to which the populations had not been exposed. In previous studies (25), populations selected on CI 13227 for greater fitness had greater fitness on L574-1 (i.e., larger initial uredinium area and growth rate and greater sporulation). This cross-adaptation of the fungus to both cultivars likely is due to the sharing of genes for resistance to $P$. triticina. Lehman et al. (23) provide evidence for a shared major gene for long latent period based on the segregation of F1, F2, and backcross progeny of a cross between CI 13227 and L574-1. Similarly, Lehman et al. (23) reported that CI 13227 and L-574-1 share at least one gene for uredinium size.

Latent period heritabilities of P. triticina on CI 13227 generally were larger than those for populations on Sw 72469-6; there is more genetic variation in latent period for fungal populations on CI 13227 than for Sw 72469-6 relative to phenotypic variation. In light of the role that genetic variation plays in the adaptation of asexual pathogen population toward partially resistance cultivars, we anticipate the resistance of CI 13227 to be relatively less durable than Sw 72469-6 when deployed under similar condi- 
tions. As described in a review by Niks and Rubiales (31), the mechanism of host resistance (e.g., prehaustorial resistance versus stoma penetration) also may influence durability (31). Although the mechanisms of resistance to P. triticina in CI 13227 and Sw 72469-6 are unknown, it is possible that they are not the same in the two cultivars and not equally durable.

In previous studies, broad-sense heritabilities of latent period for isolates of $P$. triticina on CI 13227 and Sw 72469-6 were calculated from the mean squares of analysis of variance of $\log _{10}$ mean and ranged from 0.41 to 0.49 and 0.28 to 0.53 , respectively (24). In this study, realized, broad-sense heritabilities estimates of wild-type populations on CI 13227 and Sw 72469-6 were 0.65 to 0.76 and 0.17 to 0.24 , respectively. Differences between the two studies likely are due to populations of $P$. triticina studied. In the previous study, diversity in $P$. triticina was represented by arbitrarily selected isolates from pathogen populations in Indiana and Kansas (24). The method of sampling single-uredinial isolates favored the most frequent genotypes (i.e., wild-type genotypes). In the latter study, heritability was estimated from response to selection, where populations were selected specifically for shortened latent period and, therefore, exhibited greater variation. Thus, the latter may show greater genetic variation for the latent period of $P$. triticina on partially resistant wheat cultivars.

In conclusion, the selection of wild-type populations for shortened latent period on two partially resistant cultivars resulted in populations with characteristics, not limited to latent period, that could provide greater fitness in nature. Generally, more cycles of selection had greater effects on fitness. In particular cases, the selected population on a partially resistant cultivar had values for latent period, uredinium area, and sporulation no different from that of a susceptible host-pathogen combination. Such results suggest that the variation to overcome host resistance exists in extant populations of $P$. triticina. Estimates of realized, broadsense heritability suggest that, for populations of $P$. triticina on CI 13227 , more of the variation in latent period was genetic than for populations on Sw 72469-6. Hence, CI 13227 may be more vulnerable to pathogen adaptation. Parasitic fitness, however, is a function of more that just latent period, uredinium area, and sporulation. Hence, linkage between the traits short latent period, large uredinia area, and greater sporulation to traits that decrease the parasitic fitness or traits that prevent overwintering of the fungus may increase the durability of CI 13227 or any partially resistant cultivar.

\section{ACKNOWLEDGMENTS}

This research was supported in part by Public Varieties Indiana, Inc., the Purdue Research Foundation, and Otterbein College. Purdue Agricultural Experiment Station Journal Paper No. 2007-18135.

\section{LITERATURE CITED}

1. Alexander, H. M., Groth, J. V., and Roelfs, A. P. 1985. Virulence changes in Uromyces appendiculatus after five asexual generations on a partially resistant cultivar of Phaseolus vulgarus. Phytopathology 75:449-453.

2. Bjarko, M. E., and Line, R. F. 1988. Heritability and number of genes controlling leaf rust resistance in four cultivars of wheat. Phytopathology 78:457-461.

3. Bjarko, M. E., and Line, R. F. 1988. Quantitative determination of the gene action of leaf rust resistance in four cultivars of wheat, Triticum aestivum. Phytopathology 78:451-456.

4. Broers, L. H. M., and Jacobs, T. 1989. The inheritance of host plant effect on latency period of wheat leaf rust in spring wheat. II: Number of segregating factors and evidence for transgressive segregation in F3 and F5 generations. Pages 95-105 in: Histological and Epidemiological Studies on Partial Resistance in Wheat to Leaf Rust. L. H. M. Broers and T. Jacobs, eds. Ponson \& Looijen bv, Wageningen, The Netherlands.

5. Crow, J., and Kimura, M. 1970. Page 5 in: An Introduction to Population Genetics Theory. Harper and Row, New York.

6. Eenink, A. H. 1976. Genetics of host-parasite relationships and uniform and differential resistance. Neth. J. Plant Pathol. 82:133-145.
7. Emara, Y. A., and Sidhu, G. 1974. Polygenic inheritance of aggressiveness in Ustilago hordei. Heredity 32:219-224.

8. Eyal, Z., Clifford, B. C., and Caldwell, R. M. 1968. A settling tower for quantitative inoculation of leaf blades of mature small grain plants with urediniospores. Phytopathology 58:530-531.

9. Falconer, D. S., and Mackay, T. F. C. 1996. Introduction to Quantitative Genetics, 4th ed. Longman, Inc., London.

10. Fisher, R. A. 1930. The Genetical Theory of Natural Selection. Clarendon Press, Oxford.

11. Gavinlertvatana, S., and Wilcoxson, R. D. 1978. Inheritance of slow rusting of spring wheat by Puccinia recondita f. sp. tritici and host parasite relationships. Trans. Br. Mycol. Soc. 71:413-418.

12. Hamid, A. H., Ayers, J. E., and Hill, R. R. 1982. Components of fitness attributes in Cochliobolus carbonum race 3. Phytopathology 72:11661169.

13. Hill, J. P., and Nelson, R. R. 1982. The heritability of three parasitic fitness attributes of Helminthosporium maydis race T. Phytopathology 72:525-528

14. Hill, W. G., and Caballero, A. 1992. Artificial selection experiments. Annu. Rev. Ecol. Syst. 23:287-310.

15. Jacobs, T., and Broers, L. H. M. 1989. The inheritance of host plant effect on latency period of wheat leaf rust in spring wheat. I: Estimation of gene action and number of effective factors in F1, F2 and backcross generations. Pages 79-91 in: Histological and Epidemiological Studies on Partial Resistance in Wheat to Leaf Rust. L. H. M. Broers and T. Jacobs, eds. T. Ponson \& Looijen bv, Wageningen, The Netherlands.

16. Kolmer, J. A. 1990. Seletion of virulence phenotypes in a heterogeneous, asexual population of Puccinia recondita f. sp. tritici. Phytopathology 80:1377-1381.

17. Kolmer, J. A. 1993. Selection in a heterogeneous population of Puccinia recondita f. sp. tritici. Phytopathology 83:909-914.

18. Kolmer, J. A., and Leonard, K. J. 1985. Genetic variation and selection for fertility in the fungus Cochliobolus heterostrophus. Heredity 55:335-339.

19. Kolmer, J. A., and Leonard, K. J. 1986. Genetic selection and adaptation of Cochliobolus heterostrophus to corn hosts with partial resistance. Phytopathology 76:774-777.

20. Kuhn, R. C., Ohm, H. W., and Shaner, G. E. 1980. Inheritance of slow leaf rusting resistance in Suwon 85 wheat. Crop Sci. 20:655-659.

21. Lee, T. S., and Shaner, G. 1985. Oligogenic inheritance of length of latent period in six slow leaf-rusting wheat cultivars. Phytopathology 75:636-643.

22. Lee, T. S., and Shaner, G. 1985. Transgressive segregation of length of latent period in crosses between slow leaf-rusting wheat cultivars. Phytopathology 75:643-647.

23. Lehman, J. S., Hanson, K. A., and Shaner, G. 2005. Relationship among genes conferring partial resistance to leaf rust (Puccinia triticina) in wheat lines CI 13227 and L-574-1. Phytopathology 95:198-205.

24. Lehman, J. S., and Shaner, G. 1996. Genetic variation in latent period among isolates of Puccinia recondita $\mathrm{f}$. sp. tritici on partially resistant wheat cultivars. Phytopathology 86:633-641.

25. Lehman, J. S., and Shaner, G. 1997. Selection of populations in Puccinia recondita $\mathrm{f}$. sp. tritici for shortened latent period on a partially resistant wheat cultivar. Phytopathology 87:170-176.

26. McDonald, B. A., and Linde, C. 2002. Pathogen population genetics, evolutionary potential and durable resistance. Annu. Rev. Phytopathol. 40:349-379.

27. McDonald, B. A., and McDermott, J. M. 1993. Population genetics of plant pathogenic fungi. BioScience 43:311-319.

28. McDonald, B. A., McDermott, J. M., Goodwin, S. B., and Allard, R. W. 1989. The population biology of host-pathogen interactions. Annu. Rev. Phytopathol. 27:77-94.

29. Merrick, M. J. 1976. Hybridisaton and selection for penicillin production in Aspergillus nidulans - A biometrical approach to strain improvement. In: Genetics of Industrial Microorganisms. K. D. MacDonald, ed. Academic Press, New York.

30. Milus, E. A., and Line, R. F. 1980. Characterization of resistance to leaf rust in Pacific Northwest wheats. Phytopathology 70:167-172.

31. Niks, R. E., and Rubiales, D. 2002. Potentially durable resistance mechanisms in plants to specialised fungal pathogens. Euphytica 124:201-216.

32. Ohm, H. W., and Shaner, G. E. 1976. Three components of slow leafrusting at different growth stages of wheat. Phytopathology 66:13561360.

33. Papa, K. E., Srb, A. M., and Federer, W. T. 1966. Selection for increased growth rate in inter- and intra-strain crosses of Neurospora. Heredity 21:595-613.

34. Pateman, J. A. 1955. Polygenic inheritance in Neurospora. Nature 176:1274-1275.

35. Roughgarden, J. 1979. Theory of Population Genetics and Evolutionary Ecology: An Introduction. MacMillan, New York.

36. Shaner, G. 1983. Growth of uredinia of Puccinia recondita in leaves of slow- and fast-rusting wheat cultivars. Phytopathology 73:931-935. 
37. Shaner, G., Buechley, G., and Nyquist, W. E. 1997. Inheritance of latent period of Puccinia recondita in wheat. Crop Sci. 37:748-756.

38. Shaner, G., and Finney, R. E. 1980. New sources of slow leaf-rusting resistance in wheat. Phytopathology 70:1183-1186.

39. Shaner, G., Ohm, H. W., and Finney, R. E. 1978. Response of susceptible and slow leaf-rusting wheats to infection by Puccinia recondita. Phytopathology 68:471-475.

40. Shaner, G., Stromberg, G. L., Lacy, G. H., Barker, K. R., and Pirone, T. P. 1992. Nomenclature and concepts of pathogenicity and virulence. Annu. Rev. Phytopathol. 30:47-66.
41. Simchen, G. 1966. Monokaryotic variation and haploid selection in Schizophyllum commune. Heredity 21:241-263.

42. Steel, R. G. D., Torrie, J. H., and Dickey, D. A. 1997. Principles and Procedures of Statistics: A Biometric Approach, 3rd ed. McGraw-Hill, New York.

43. Tervet, I. W., Rawson, A. J., Cherry, E., and Saxon, R. B. 1950. A method for the collection of microscopic particles. Phytopathology 41:282-285.

44. Wilson, J., and Shaner, G. 1989. Individual and cumulative effects of long latent period and low infection type reactions to Puccinia recondita in triticale. Phytopathology 79:101-108. 